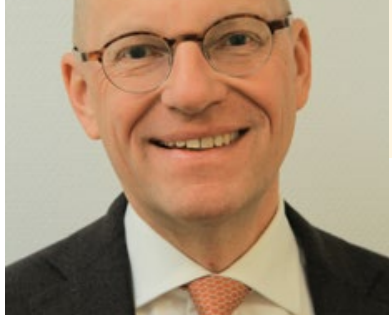

\title{
Geriatrie braucht Priorisierung zum Wohle der Patienten
}

S ehr alt zu werden, ist heutzutage eher Regel denn Ausnahme. Ernährung, Bewegung, soziale Integration und psychische Gesundheit sind bekannte Faktoren, die ein "gesundes Altern“ ermöglichen und damit Komorbiditäten verhindern. Eine Komorbidität liegt dann vor, wenn neben einer Indexerkrankung eine oder gar mehrere diagnostisch abgrenzbare Krankheitsbilder vorliegen. Dabei können Komorbiditäten im Sinne einer Folgeerkrankung auch ursächlich mit der Grunderkrankung zusammenhängen.

Die Evidenz-basierte Medizin (EbM) in ihrer aktuellen Auslegung ist für Betagte und Hochbetagte kritisch zu sehen, da sie erstens meist auf einer Monopathologie basiert und zweitens das Überleben als primären Endpunkt wertet. Dies hängt auch damit zusammen, dass sich die heutige EbM primär auf wissenschaftliche Studien konzentriert, und die bereits in der Erstbeschreibung der EbM wichtigen Säulen „Erfahrung des Arztes“ sowie „Patientenpräferenzen“ als eher sekundär wertet. Die Erfahrungen des Arztes und des geriatrischen, multiprofessionellen Teams sind gerade aufgrund der Multimorbidität, respektive bei Komorbidität zu einer Indexerkrankung, eminent wichtig. Dazu gehört auch, unerreichbare Ziele anzusprechen, wie beispielsweise das der Reversibilität von Demenzerkrankungen.

Typisch für Multimorbidität aber auch für die meisten Komorbiditäten ist, dass für eine zielführende Diagnostik und Therapie das Zusammenspiel verschiedener medizinischer Fachdisziplinen wie auch multiprofessioneller Teams notwendig ist. Chronische Krankheiten - inklusive Komorbiditäten - zeigen dabei folgende Eigenschaften:

_Die zeitliche Dauer ist nicht limitiert, _die Aussicht auf Heilung ist sehr beschränkt, _-der Krankheitsverlauf ist undulierend, _die Ätiologie der Krankheit ist meist komplex und nicht eindeutig (z. B. beim Sturzsyndrom),

-körperliche und psychische Komponenten überlappen sich,

_ das Leben mit Unsicherheit und Mehrdeutigkeit stellt spezifische Herausforderungen sowohl für die Betroffenen als auch für die Pflegenden,

_ die Folgen der chronischen Krankheit sind vielschichtig und häufig auch weitreichend für die soziale Teilhabe.

Einige dieser Problemfelder werden auf dem nächstjährigen Kongress der Deutschen Gesellschaft für Innere Medizin (DGIM) im April 2018 in Mannheim im Zentrum stehen, beispielsweise "Chronische Krankheiten und EbM“, „Multimorbidität und Polypharmazie“, „Internisten als Teil interdisziplinärer Teams“ oder „Rehabilitation als Bindeglied zu sozialer Teilhabe“.

Auch im vorliegenden Heft von Geriatrie-Report werden wichtige Themen der Altersmedizin besprochen. „Herausgepickt" seien hier mehrere Beiträge zum Thema „Altersmedizin: Ist das, was machbar ist, auch sinnvoll?" (S. 8, 12, 16, 28). Hierbei gibt es wichtige Bezüge zur Initiative der Deutschen Gesellschaft für Innere Medizin (DGIM) „Klug entscheiden".

Bei der Betreuung der uns anbefohlenen betagten und hochbetagten, multimorbiden Menschen müssen wir in deren Sinne eine Priorisierung vornehmen. Medizinethisch ist dies weit entfernt von Rationierung, sondern eine mit dem Patienten abgestimmte Zielsetzung - eigentlich ein integraler Teil der EbM. „Patient-valued oder patient-centered care" ist hier das Stichwort.

Ich wünsche Ihnen eine spannende Lektüre unseres Geriatrie-Reports.

Cornel Sieber 